\title{
Efficient inhibition of hepatitis B virus replication by hepatitis delta virus ribozymes delivered by targeting retrovirus
}

\author{
Chuan-Xi Wang 1,2, Yan-Qin Lu², Peng Qi2, Long-Hua Chen*1 and Jin-Xiang Han*2
}

\begin{abstract}
Background: Hepatitis delta virus (HDV) ribozyme is an attractive molecular tool that can specifically recognize and catalyze the self-cleavage of the viral RNA phosphodiester backbone. However, a major obstacle in the medical application of the HDV ribozyme is the lack of specificity in the delivery of the ribozyme to defined target cells.

Results: The objective of this study was to determine whether retroviral vectors can deliver the HDV ribozyme into the target cells and to elucidate whether HDV ribozyme plays a role in hepatitis B virus (HBV) replication. In our study, the transduction of helper-free pseudotyped retrovirus, which showed a broad host range, in human hepatoma cells was performed under 2 conditions, that is, in the presence of polymerized human serum albumin (pHSA) and in the absence of pHSA. The transduction ability in the presence of pHSA was higher than in the absence of pHSA. Moreover, $\mathrm{HBsAg}$ and HBeAg levels after transductions with pHSA were significantly lower than those in the absence of pHSA, thus indicating that the recombinant retrovirus had HBV-specific cleavage activity and targeted HepG2215 cells.
\end{abstract}

Conclusions: These data suggest that this system provides a new approach for targeting hepatocytes and has a great potential in gene therapy for HBV infection.

\section{Introduction}

Hepatitis B virus (HBV) causes acute and chronic infections of the liver. Acute infections can cause serious illnesses and lead to fatal fulminant hepatitis in approximately $0.5 \%$ of the patients. Chronic infections may also induce serious consequences leading to untreatable hepatocellular carcinoma (HCC) in nearly $25 \%$ of the patients. The number of deaths attributed to hepatocellular carcinoma caused by HBV infection in the world probably exceeds 1 million per year [1-3]. Moreover, the various treatments for chronic infections have had only limited success [4].

The long-term effects of the recent advanced techniques employed to eliminate the virus, including therapy with nucleoside analogs and other virus-replication inhibitors [5,6], are yet to be determined. Since HBV reverse transcriptase lacks proofreading function, the

\footnotetext{
*Correspondence: flchenlh@gmail.com, hanjx69@126.com

1 Department of Radiation Oncology, Nanfang Hospital, Southern Medical University, Guangzhou 510515, Guangdong Province, PR China

2 Shandong Medicinal and Biotechnology Center, Shandong Academy of

Medical Sciences, 89 Jingshi Road, Jinan 250062, Shandong Province, PR China
}

virus shows rapid mutagenesis thus creating a large number of variants, some of which show resistance to antiviral drugs. This phenomenon is responsible for the low efficacy of the current drugs and the high rates of drug resistance $[7,8]$. Therefore, there is an urgent need to develop new anti-HBV drugs.

A ribozyme $(\mathrm{Rz})$ is a small RNA molecule that can act as an enzyme. Ribozymes catalyze the cleavage of specific mRNAs in a sequence-specific manner; therefore, they are attractive therapeutic tools for the inactivation of both viral RNA and mRNAs associated with human diseases $[9,10]$. The ribozyme found in the genomic and antigenomic RNAs of the hepatitis delta virus (HDV) adopts a novel structural motif that is distinct from the hammerhead and hairpin motifs of ribozymes found predominantly in the plant pathogenic RNAs [11,12]. This HDV ribozyme shows a unique natural ability to function in human cells.

Viruses have been used to introduce exogenous DNA sequences into target cells in many gene-therapy strategies for treating genetic diseases, including cancer. Among the various viral vectors engineered for this pur- 
pose, those based on retroviruses are the best understood and the most widely used $[13,14]$. The genomes of the viral vectors integrate stably into the host cell DNA, thereby allowing long-term expression of the inserted therapeutic genes in the host cells. The processes of virus entry and genome integration do not require viral protein synthesis. Therefore, all viral genes in the vector genome can be replaced with exogenous sequences. However, a major obstacle to the medical application of such vectors is the lack of specificity in gene delivery to defined target cells.

In the present study, we designed HDV ribozymes to cleave HBV-RNA (ayw subtype). The cleavage site was selected using structural data obtained by computerassisted methods [15]. The use of bioinformatics tools coupled to biochemical assays; RNase $\mathrm{H}$ hydrolysis with a pool of oligonucleotides; and cleavage assays with a pool of ribozymes. Potential $\mathrm{Rz}$ target site was identified by these procedures and the substrate RNA contained HBV core region. Rz shows site-specific cleavage of HBV RNA at certain sites under appropriate conditions in vitro. However, the intracellular conditions and the factors that influence ribozyme activity are far more complicated than the conditions in the extracellular environment; therefore, there is no data describing whether the HDV ribozyme can cleave HBV mRNA in vivo. In this study, the DNA encoding HDV ribozyme was amplified and cloned in the retroviral vector pMSCV/U6 (Clontech), and the resultant recombinant vector was named pRz. Using the calcium phosphate-mediated DNA-transfection technique, 293T cells were transfected with pRz, Moloney murine leukemia virus (Mo-MLV), Gag-Pol expression plasmid (pGAG-POL), and the chimeric envelope expression plasmid (pENV-preS2) [16,17], which contain the hepatitis B virus PreS2 peptide fused to aa +1 at the $\mathrm{N}$ terminus of Env. At $48 \mathrm{~h}$ post-transfection, we obtained helper-free retrovirus stocks with titers of 2.9-4 $\times 10^{4} \mathrm{cfu} / \mathrm{ml}$, and these stocks were used to infect HepG2215 cells. The recombinant retrovirus carrying the HDV ribozyme could bind to hepatocytes in the presence of polymeric human serum albumin and specifically cleave the HBV mRNA.

\section{Results}

\section{Generation of Retrovirus Stocks}

In conjunction with a retroviral vector, $293 \mathrm{~T}$ cells yielded infectious, replication-incompetent retrovirus that could be used to introduce a gene of interest into a wide variety of mammalian cell types in vitro or in vivo. The transfection of 293T cells with pRz recon, Moloney murine leukemia virus (MoMLV), Gag-Pol expression plasmid (pGAG-POL), and the chimeric envelope expression plasmids--pENV-preS2 or pENV, which encode HBV preS2-Env chimeric envelope and wild-type ecotropic envelope, respectively, produced wild-type retrovirus stocks (titer, $0.9 \times 10^{6} \mathrm{cfu} / \mathrm{ml}$ ) and pseudotyped retrovirus stocks (titers, $2.9-4 \times 10^{4} \mathrm{cfu} / \mathrm{ml}$ ). The undiluted viral supernatants were used to infect HepG2215 cells.

\section{Analysis of Helper Virus}

We examined the supernatants from the transfected 293T cells to detect contaminating replication-competent viruses or helper viruses. NIH 3T3 cells were infected with undiluted supernatants of the retroviral stocks, and these cells were then passaged for 4 weeks to allow virus propagation. During each passage, the culture supernatants were harvested, filtered, and used to infect fresh NIH 3T3 cells. These cells were selected in puromycin for up to 10 days to detect the presence of replication-competent virus harboring the pur gene. No puromycin-resistant colonies were detected at 10 days post-infection.

\section{Inhibition of HBsAg and HBeAg in HepG2215 Cells}

The culture medium was analyzed using enzyme linked immunosorbent assay to determine the $\mathrm{HBsAg}$ and $\mathrm{HBeAg}$ concentrations. Figure $1 \mathrm{~A}$ shows that the pHSAmediated HDV ribozyme transfection showed more significant inhibition of HBsAg-expression than did transfection without pHSA mediation; the inhibitory effects were calculated with reference to the negative control RzNC (Rz with pHSA vs. Rz without pHSA, $P<0.001$ ). In comparison with RzNC, $\mathrm{Rz}$ and pHSA caused a 78.7\% decrease in the extracellular $\mathrm{HBeAg}$ levels at $48 \mathrm{~h}$ after treatment; however, in the absence of pHSA, the corresponding value was only $17.3 \%(\mathrm{Rz}$ with $\mathrm{pHSA}$ vs. $\mathrm{Rz}$ without pHSA, $P=0.001$ )(Figure 1B).

\section{Inhibition of HBV replication in HepG2215 Cells}

Cultures with and without retroviruses were maintained at $37^{\circ} \mathrm{C}$ for $48 \mathrm{~h}$ and then assessed for the presence of viral DNA in cell lysates. HBV DNA was analyzed quantitatively, and the relative rate of HBV replication was determined. HBV DNA was analyzed using real-time PCR. As shown in Figure 2, in comparison to the corresponding values for the negative control $\mathrm{Rz}$, the number of HBV DNA copies per ml decreased to $2.16 \times 10^{6}$ copies $/ \mathrm{ml}$ after ribozyme treatment with pHSA and to $7.26 \times 10^{6}$ copies $/ \mathrm{ml}$ after ribozyme treatment without pHSA (Rz without pHSA vs. Rz with pHSA, $P<0.001$ ).

\section{Discussion}

The currently available viral vectors for gene therapy lack host-cell specificity. During the past few years, a series of different targeting strategies, including cross-linking of MLV retroviral vectors to target cells using an antibody bridge $[18,19]$ and genetic modification of Mo-MLV Env by the addition of single-chain antibody variable fragments [20], have been developed to modify the retroviral 


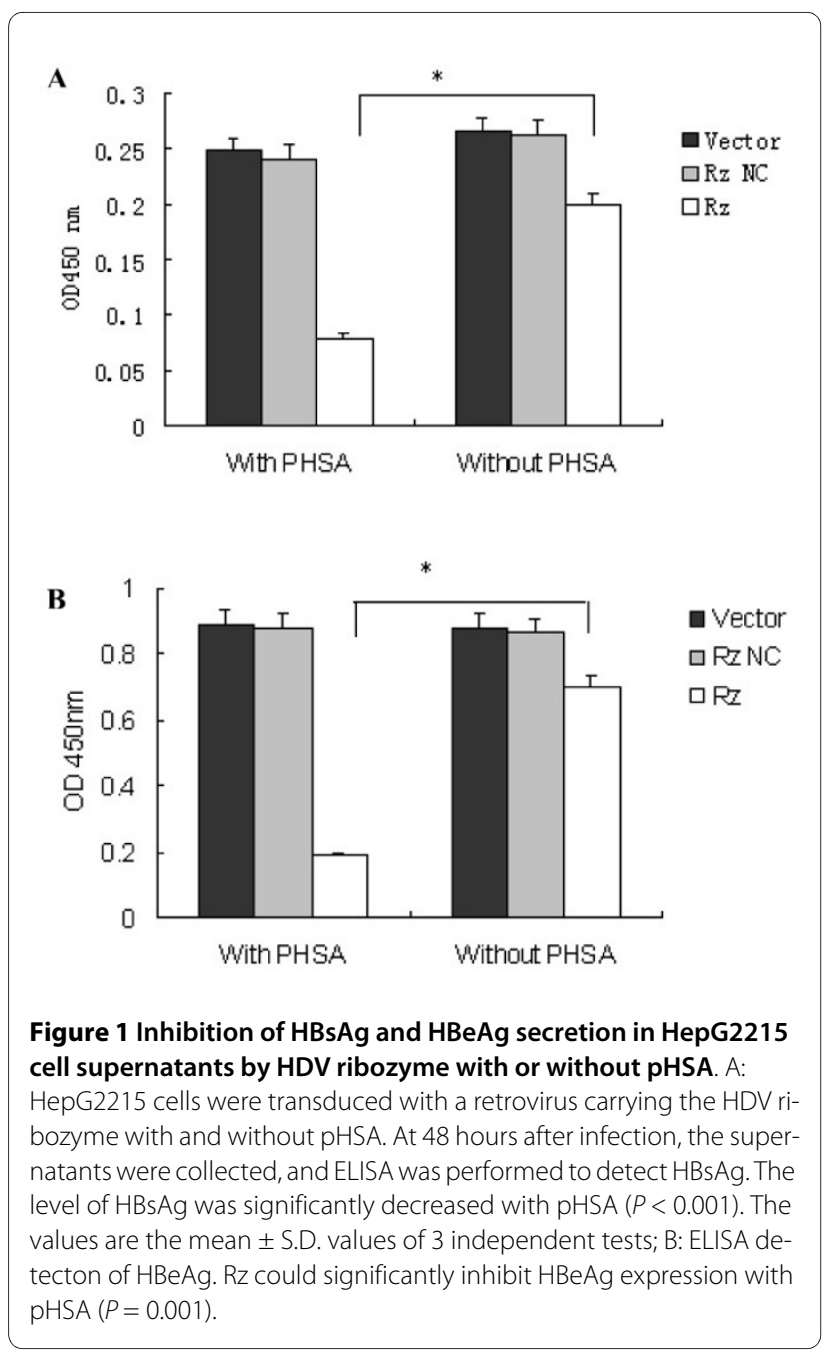

envelopes for broad host range. However, such attempts to redirect retroviruses to specific cells by attaching additional cell-binding ligands to the ecotropic MLV Env have met with little success so far. The HBV envelope is composed of 3 related surface (S) proteins. The preS2 mRNA

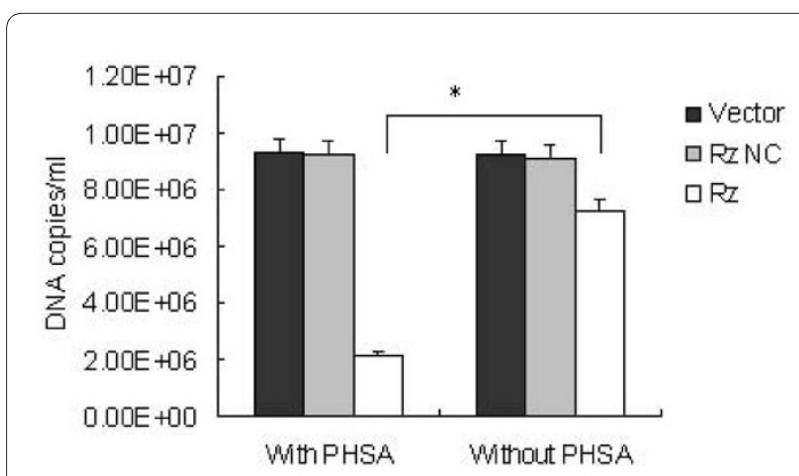

Figure 2 HBV DNA copies in HepG2215 cells. HBV DNA was extracted and real-time PCR was performed $(p<0.001)$. encodes albumin receptors, which bind to pHSA and mediate viral attachment to the hepatocytes [21]. In our previous study, we had constructed a chimeric envelope expression plasmid pENV-preS2, which express the HBV preS2 peptide fused to aa +1 at the $\mathrm{N}$-terminus of ENV protein. After cotransfection of pGAG-POL, pRz, and chimeric env plasmids into the 293T cells, retrovirus stocks were obtained at $48 \mathrm{~h}$ post-transfection. These pseudotyped vectors infected the normal host NIH 3T3 cells although the efficiency of infection was lesser than that of the virions with the wild-type ecotropic MoMLV envelope. In addition, the transduction ability of the pseudotyped viruses in human hepatoma cells was higher in the presence of pHSA than in the absence of pHSA, thereby indicating that pHSA could attach to the hepatocyte membranes Thus, pHSA receptor acts as an "intermediate ligand" [16].

Since no helper virus could be detected after 10 days post-infection, we deduced that the viral stocks obtained in this study did not contain helper viruses. Here, we report a significant reduction in the intracellular HBV DNA concentration in HepG2215 cells, which markedly decreased after 48 hours of incubation with Rz. Similar findings have been reported in previous studies $[22,23]$.

The $\mathrm{Rz}$ used in this experiment is known to show high cleavage activity in HBV mRNA. Initially, we tried to design a series of trans-HDV ribozymes to cleave HBV mRNA [15]. The substrate RNAs were RNA sequences from the $\mathrm{C}$ region of the HBV genome. The result show that under the optimized cleavage conditions, ribozyme specifically cleaved the mRNA of the HBV core gene; further, at $90 \mathrm{~min}$, the percentage of the cleaved substrates increased with time: up to $53 \%$.

The HDV ribozymes are potentially attractive tools for directed RNA degradation [24]. These ribozymes are naturally active in human cells, and at physiological $\mathrm{Mg}^{2+}$ ion concentrations, they show the highest cleavage rates among all known ribozymes [25-27].

In this study, we have shown that the retrovirus-based $\mathrm{Rz}$ could significantly inhibit HBV antigen expression. Our results show that the extracellular HBsAg and HBeAg levels after treatment with HDV ribozyme were lower than those after treatment with the negative control ribozyme. $\mathrm{HBsAg}$ and $\mathrm{HBeAg}$ are translated from preS/S mRNA and precore mRNA, respectively. Although $\mathrm{HBeAg}$ is translated from precore mRNA, the pre $\mathrm{C}$ and $\mathrm{C}$ regions are in the same open reading frame (ORF). In addition, the entire 2.1-kb mRNA encoding HBsAg comprises overlapping reading frames, and this sequence is contained within the $3.5-\mathrm{kb}$ mRNA sequence encoding HBeAg. Since Rz could specifically cleave the C region RNA, HBeAg expression was suppressed; thus, the HBeAg and HBsAg levels decreased. We also observed downregulation of the number of HBV DNA copies/ml 
after incubation with $\mathrm{Rz}$-containing retrovirus comparing both ribozyme negative control and empty vector, instead of intrinsic effect of transduction. The Rz inhibited HBV pregenomic RNA, which is reverse transcribed to viral DNA; therefore, HBV replication is impeded. Hence, $\mathrm{HBV}$ is assumed to be susceptible to $\mathrm{Rz}$ at both the posttranscription and replication levels. These results are in accordance with those obtained by $\mathrm{Li}$ et al [28], who found that a dual-shRNA expression vector (AAV-157i/ 1694i), which coexpressed 2 shRNAs, targeted the $S$ and $\mathrm{X}$ genes of $\mathrm{HBV}$ and reduced the levels of $\mathrm{HBsAg}$, HBeAg, and HBV DNA by $87 \% \pm 4 \%, 80.3 \% \pm 2.6 \%$, and $86.2 \% \pm 7 \%$ respectively, at 8 days post-transduction. In a mouse model of prophylactic treatment, $\mathrm{HBsAg}$ and $\mathrm{HBeAg}$ were reduced to undetectable levels, and the serum HBV DNA level showed at least a 100-fold reduction.

\section{Conclusions}

The HBV-specific HDV ribozyme can catalyze sequencespecific HBV mRNA degradation and suppress HBV replication. The retrovirus carrying $\mathrm{Rz}$ has the potential for use in gene therapy for HBV infection.

\section{Materials and methods \\ Cell Culture}

The following cell lines were grown in Dulbecco's Modified Eagle's Medium (DMEM, Gibco, USA) supplemented with $10 \%$ fetal bovine serum (Gibco, USA): $293 \mathrm{~T}$ (ATCC CRL-11268), NIH 3T3 (ATCC CRL-1658), HepG2215, and human embryonic kidney cells (HEK) (ATCC CRL-1573). All cells were cultured in $5 \% \mathrm{CO}_{2}$ at $37^{\circ} \mathrm{C}$.

\section{Construction of the pRz Recon}

The HDV ribozyme targeting the HBV core-gene sequence was synthesized. The sequences of ribozyme were: Rz sense: 5'-CGCGGGCCCAGCTRGGGTCCACCTCCTCGCGGTGGTGGCTGGG-3' (Apa I) and Rz antisense:5'-CGCGAATTCTCGACCCAGCCACCACCGCGAGGAGGTGGACCCA-3' (EcoR I). Then, this sequence was cloned into the pGEM-T vector (Promega) and subcloned in the multiple cloning sites of the retroviral plasmid pMSCV/U6, which was digested with the same restriction enzymes (Figure 3); the resultant recombinant recon was called the $\mathrm{pRz}$ recon. The ribozyme of a random sequence unrelated to $\mathrm{HBV}$ was amplified with PCR and cloned into pMSCV/U6 to obtain pRzNC, which was considered as the negative control. All ribozyme sequences were confirmed by sequencing.

\section{Retrovirus packaging, Infection, and Determination of Viral} Titer

The 293T cells were transfected by overnight calcium phosphate treatment on $10-\mathrm{cm}$ dishes seeded on the previous day, which yielded a maximum of $70 \%$ confluence/

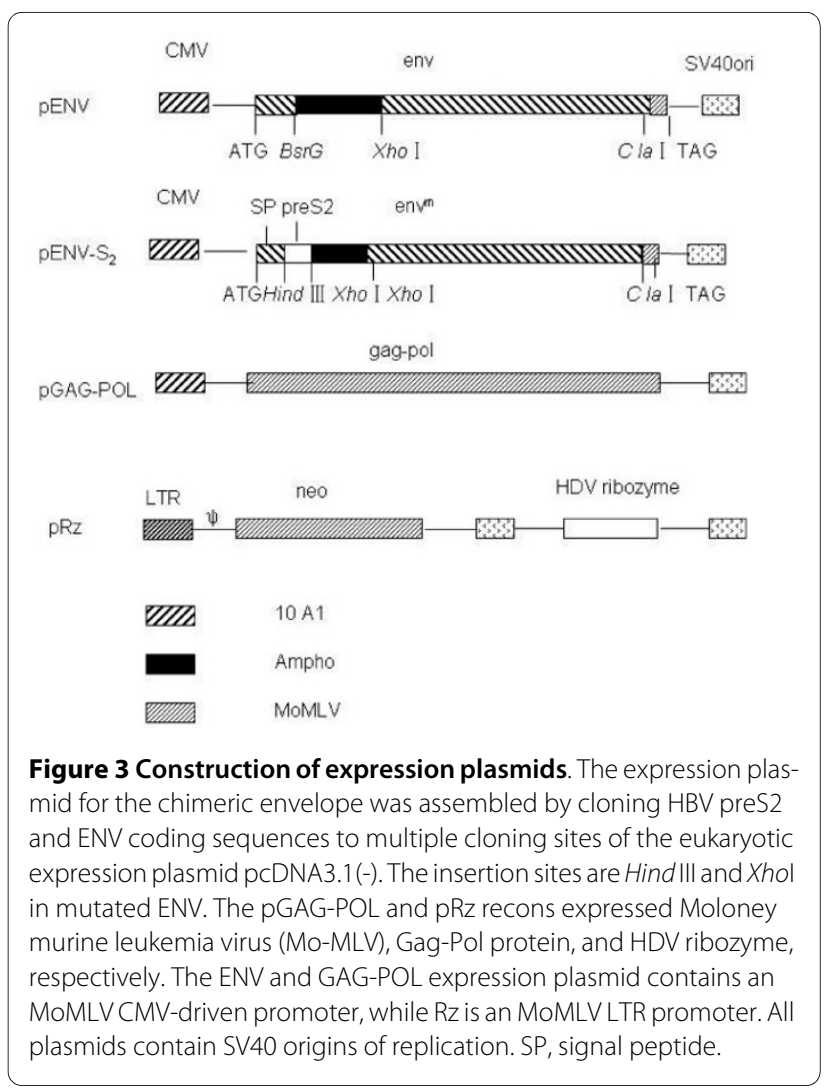

plate on the day of transfection. Retroviruses were generated by cotransfection of $293 \mathrm{~T}$ cells with $10 \mu \mathrm{g}$ of pRz, 20 $\mu \mathrm{g}$ of pGAG-POL, and $10 \mu \mathrm{g}$ of pENV-preS2. The transfected cells were incubated for 15-17 $\mathrm{h}$ after which the medium was replaced with $5 \mathrm{ml}$ of fresh medium. The viral supernatants were harvested $48 \mathrm{~h}$ after transfection, filtered through $0.45 \mu \mathrm{m}$ filters, and titered by serial dilution on NIH 3T3 cells [29].

$\mathrm{NIH} 3 \mathrm{~T} 3$ cells were plated onto 6 -well culture dishes at a density of $1-2 \times 10^{5}$ cells/well on the day before infection. A fixed volume $(1 \mathrm{ml})$ of the undiluted supernatant and $8 \mu \mathrm{g} / \mathrm{ml}$ polybrene (Sigma) was added to each well, and the wells were incubated for $2 \mathrm{~h}$, which was followed by the addition of $1 \mathrm{ml}$ of fresh medium. Twenty four hours later, the cells were diluted (1:4) and seeded onto 5$\mathrm{cm}$ dishes. The viral titer was determined by selection with $800 \mu \mathrm{g} / \mathrm{ml}$ puromycin. The medium was replaced every 3 or 4 days, and the resistant colonies were counted $10-15$ days post-infection by fixing and staining with $0.3 \%$ crystal violet in $70 \%$ methanol. The viral titer was determined by multiplying the total number of puromycinresistant colonies by 2 , which was performed to account for the 2-fold increase in the cells during the infection period, and by considering the 1:4 dilutions.

\section{Helper Virus Assays}

Transfections in 293T cells were performed using the protocols described above, and undiluted viral supernatants were used to infect NIH 3T3 cells. All infections 
were performed in the presence of $8 \mu \mathrm{g} / \mathrm{ml}$ polybrene. The NIH 3T3 cells were passaged every 4-5 days, and the culture supernatants were filtered and used to infect fresh NIH 3T3 cells. These cells were selected in puromycin for up to 10-15 days and used to assay for the presence of replication-competent viruses.

\section{Analysis of Ribozyme in NIH 3T3 Cells}

Transfection and infection were performed on $293 \mathrm{~T}$ and NIH 3T3 cells, respectively, by using the methods described above. Genomic DNA from infected cells was extracted using the DNeasy kit (QIAGEN) according to the manufacturer's instructions. The ribozyme was detected by PCR. The primers P1 and P2 were used as described above.

\section{Enzyme linked immunosorbent assay (ELISA) and Real-time PCR Assays}

On the day before infection, HepG2215 cells were seeded in 24-well tissue culture dishes at a density of $3 \times 10^{4}$ cells/well. A total of $250 \mu \mathrm{l}$ of the undiluted supernatants was added to each well along with $8 \mu \mathrm{g} / \mathrm{ml}$ of polybrene and incubated for $2 \mathrm{~h}$; the incubation was followed by the addition of $1 \mathrm{ml}$ of fresh medium. Each experiment was performed in triplicate. For pHSA-mediated experiments, pHSA $(5 \mu \mathrm{g} / \mathrm{ml})$ was added to the culture medium, which was followed by incubation at $4^{\circ} \mathrm{C}$ for $4 \mathrm{~h}$ and viral infection. The supernatants and cells were harvested once everyday for 4 days. The levels of hepatitis $B$ surface antigen (HBsAg) and hepatitis $B$ e antigen $(\mathrm{HBeAg})$ were determined using licensed ELISA kits (Shanghai Rongsheng Biotech Co. Ltd.)according to the manufacturer's instructions. Genomic DNA was extracted from the infected cells by using the QIAGEN DNeasy kit. Real-time (RT) quantitative PCR was performed using the following gene-specific primers: P3, 5'-AGAATCCTCACAATACCGCAGAGT-3'; P4, - 5'CACACGGTAGTTCCCCCTAGAA-3'; and probe: P5, 5'-FAM-AGACTCGTGGTGGACTTCTCTCAAT-

TAMRA-3' [30]. The standards were obtained using the above primers. For RT-PCR, the $20-\mu \mathrm{L}$ reaction mixture contained $1 \mu \mathrm{L}$ of genomic DNA or standard DNA, $2 \mu \mathrm{L}$ of $10 \times$ PCR reaction buffer, $20 \mathrm{mmol}$ of each dNTP, 5 $\mu \mathrm{mol}$ of the primers, $2.5 \mu \mathrm{mol}$ of the probe, and $1 \mathrm{U}$ of hot-start DNA polymerase (TAKARA). All amplification reactions were performed in triplicate. The following PCR cycle was used: initial denaturation at $95^{\circ} \mathrm{C}$ for $30 \mathrm{~s}$ followed by 40 cycles of denaturation at $95^{\circ} \mathrm{C}$ for $5 \mathrm{~s}$ and annealing at $60^{\circ} \mathrm{C}$ for $34 \mathrm{~s}$. In each cycle, fluorescence readings were recorded at $60^{\circ} \mathrm{C}$. Real-time PCR was done on the 7500 Real-Time PCR System (Applied Biosystems).

\section{Statistical Analysis}

Paired-samples $t$ test was performed using SPSS 17.0. The differences were considered statistically significant when $P$ values were less than 0.05 .

\section{List of abbreviations}

HCC: hepatocellular carcinoma; HBV: Hepatitis B virus; HBsAg: hepatitis B surface antigen; HBeAg: hepatitis B e antigen; Rz: ribozyme; HDV: hepatitis delta virus; MoMLV: Moloney murine leukemia virus; Env: chimeric envelope; pHSA: polymerized human serum albumin; ELISA: Enzyme linked immunosorbent assay.

\section{Competing interests}

The authors declare that they have no competing interests.

\section{Authors' contributions}

CXW carried out most of the studies and drafted the manuscript. YQL and PQ participated parts of the studies and writing. LHC and JXH provided consultation and preparation of the final report. All authors read and approved the final manuscript.

\section{Acknowledgements}

This work was supported in part by the National Natural Science Foundation of China (No. 30371328, 30772530); the National High Technology Research and Development Program of China (863 Program, No2007AA021004); and the Shandong Health Department program of China (No. 2007QW032).

\section{Author Details}

'Department of Radiation Oncology, Nanfang Hospital, Southern Medical University, Guangzhou 510515, Guangdong Province, PR China and 2Shandong Medicinal and Biotechnology Center, Shandong Academy of Medical Sciences, 89 Jingshi Road, Jinan 250062, Shandong Province, PR China

Received: 20 December 2009 Accepted: 17 March 2010

Published: 17 March 2010

\section{References}

1. Kim WR: Epidemiology of hepatitis B in the United States. Hepatology 2009, 49:S28-34.

2. McMahon BJ: The natural history of chronic hepatitis B virus infection. Hepatology 2009, 49:S45-55.

3. Tan A, Yeh SH, Liu CJ, Cheung C, Chen PJ: Viral hepatocarcinogenesis: from infection to cancer. Liver Int 2008, 28:175-188.

4. Shamliyan TA, MacDonald R, Shaukat A, Taylor BC, Yuan JM, Johnson JR, Tacklind J, Rutks I, Kane RL, Wilt TJ: Antiviral therapy for adults with chronic hepatitis B: a systematic review for a National Institutes of Health Consensus Development Conference. Ann Intern Med 2009, 150:111-124.

5. Papadopoulos VP, Chrysagis DN, Protopapas AN, Goulis IG, Dimitriadis GT, Mimidis KP: Peginterferon alfa-2b as monotherapy or in combination with lamivudine in patients with $\mathrm{HBeAg}$-negative chronic hepatitis $\mathrm{B}: \mathrm{a}$ randomised study. Med Sci Monit 2009, 15:CR56-61.

6. Wilt TJ, Shamliyan T, Shaukat A, Taylor BC, MacDonald R, Yuan JM, Johnson JR, Tacklind J, Rutks I, Kane RL: Management of chronic hepatitis B. Evid Rep Technol Assess (Full Rep) 2008:1-671.

7. Mauss S, Wedemeyer H: Treatment of chronic hepatitis B and the implications of viral resistance to therapy. Expert Rev Anti Infect Ther 2008, 6:191-199.

8. Locarnini S, Warner N: Major causes of antiviral drug resistance and implications for treatment of hepatitis $B$ virus monoinfection and coinfection with HIV. Antivir Ther 2007, 12(Suppl 3):H15-23.

9. Kim DE, Joyce GF: Cross-catalytic replication of an RNA ligase ribozyme. Chem Biol 2004, 11:1505-1512. 
10. Tobe S, Heams T, Vergne J, Herve G, Maurel MC: The catalytic mechanism of hairpin ribozyme studied by hydrostatic pressure. Nucleic Acids Res 2005, 33:2557-2564

11. Perrotta AT, Been MD: A pseudoknot-like structure required for efficient self-cleavage of hepatitis delta virus RNA. Nature 1991, 350:434-436.

12. Cochrane JC, Strobel SA: Catalytic strategies of self-cleaving ribozymes. Acc Chem Res 2008, 41:1027-1035.

13. Tai CK, Kasahara N: Replication-competent retrovirus vectors for cancer gene therapy. Front Biosci 2008, 13:3083-3095.

14. Dalba C, Klatzmann D, Logg CR, Kasahara N: Beyond oncolytic virotherapy: replication-competent retrovirus vectors for selective and stable transduction of tumors. Curr Gene Ther 2005, 5:655-667.

15. Bergeron LJ, Perreault JP: Development and comparison of procedures for the selection of delta ribozyme cleavage sites within the hepatitis $B$ virus. Nucleic Acids Res 2002, 30:4682-4691.

16. Qi P, Han J, Lu Y, Wang C, Zhu B: A transient three-plasmid expression system for the production of hepatocytes targeting retroviral vectors. Acta Biochim Biophys Sin (Shanghai) 2007, 39:567-574.

17. Miller $A D$, Chen F: Retrovirus packaging cells based on $10 \mathrm{~A} 1$ murine leukemia virus for production of vectors that use multiple receptors for cell entry. J Virol 1996, 70:5564-5571.

18. Goud B, Legrain P, Buttin G: Antibody-mediated binding of a murine ecotropic Moloney retroviral vector to human cells allows internalization but not the establishment of the proviral state. Virology 1988, 163:251-254.

19. Roux $P$, Jeanteur $P$, Piechaczyk M: A versatile and potentially general approach to the targeting of specific cell types by retroviruses: application to the infection of human cells by means of major histocompatibility complex class I and class II antigens by mouse ecotropic murine leukemia virus-derived viruses. Proc Natl Acad Sci USA 1989, 86:9079-9083.

20. Lorimer IA, Lavictoire SJ: Targeting retrovirus to cancer cells expressing a mutant EGF receptor by insertion of a single chain antibody variable domain in the envelope glycoprotein receptor binding lobe. $J$ Immunol Methods 2000, 237:147-157.

21. Ohnuma H, Takahashi K, Kishimoto S, Machida A, Imai M, Mishiro S, Usuda S, Oda K, Nakamura T, Miyakawa Y, et al:: Large hepatitis B surface antigen polypeptides of Dane particles with the receptor for polymerized human serum albumin. Gastroenterology 1986, 90:695-701.

22. Citti L, Rainaldi G: Synthetic hammerhead ribozymes as therapeutic tools to control disease genes. Curr Gene Ther 2005, 5:11-24.

23. Tedeschi L, Lande C, Cecchettini A, Citti L: Hammerhead ribozymes in therapeutic target discovery and validation. Drug Discov Today 2009, 14:776-783.

24. Sefcikova J, Krasovska MV, Sponer J, Walter NG: The genomic HDV ribozyme utilizes a previously unnoticed U-turn motif to accomplish fast site-specific catalysis. Nucleic Acids Res 2007, 35:1933-1946.

25. Chen JH, Gong B, Bevilacqua PC, Carey PR, Golden BL: A catalytic metal ion interacts with the cleavage Site G.U wobble in the HDV ribozyme. Biochemistry 2009, 48:1498-1507.

26. Tinsley RA, Harris DA, Walter NG: Magnesium dependence of the amplified conformational switch in the trans-acting hepatitis delta virus ribozyme. Biochemistry 2004, 43:8935-8945.

27. Chadalavada DM, Cerrone-Szakal AL, Bevilacqua PC: Wild-type is the optimal sequence of the HDV ribozyme under cotranscriptional conditions. RNA 2007, 13:2189-2201.

28. Li Z, He ML, Yao H, Dong QM, Chen YC, Chan CY, Zheng BJ, Yuen KY, Peng $Y$, Sun $Q$, et al: Inhibition of HBV replication and gene expression in vitro and in vivo with a single AAV vector delivering two shRNA molecules. BMB Rep 2009, 42:59-64.

29. Gong YS, Zhang KL, Jiang XG, Wang ZW, Sun ZQ, Cai J: Retroviral gene transfer of tissue-type plasminogen activator targets thrombolysis in vitro and in vivo. Gene Ther 2007, 14:1537-1542.

30. Lu YQ, Han JX, Qi P, Xu W, Zu YH, Zhu B: Rapid quantification of hepatitis $B$ virus DNA by real-time $P C R$ using efficient TaqMan probe and extraction of virus DNA. World J Gastroenterol 2006, 12:7365-7370.

doi: $10.1186 / 1743-422 X-7-61$

Cite this article as: Wang et al., Efficient inhibition of hepatitis B virus replication by hepatitis delta virus ribozymes delivered by targeting retrovirus Virology Journal 2010, 7:61

\section{Submit your next manuscript to BioMed Centra and take full advantage of:}

- Convenient online submission

- Thorough peer review

- No space constraints or color figure charges

- Immediate publication on acceptance

- Inclusion in PubMed, CAS, Scopus and Google Scholar

- Research which is freely available for redistribution

Submit your manuscript at www.biomedcentral.com/submit
C Biomed Central 